\title{
Genetic Diversity in the Camel Tick Hyalomma dromedarii (Acari: Ixodidae) Based on Mitochondrial Cytochrome c Oxidase Subunit I (COI) and Randomly Amplified Polymorphic DNA Polymerase Chain Reaction (RAPD-PCR)
}

\author{
Mohammad Ali Al-Deeb', Mohamed Rizk Enan",2 \\ ${ }^{1}$ Biology Department, United Arab Emirates University, Al-Ain, UAE \\ ${ }^{2}$ Agricultural Research Center, Agricultural Genetic Engineering Research Institute, Giza, Egypt \\ Email: m_aldeeb@uaeu.ac.ae
}

How to cite this paper: Al-Deeb, M.A. and Enan, M.R. (2018) Genetic Diversity in the Camel Tick Hyalomma dromedarii (Acari: Ixodidae) Based on Mitochondrial Cytochrome c Oxidase Subunit I (COI) and Randomly Amplified Polymorphic DNA Polymerase Chain Reaction (RAPD-PCR). Advances in Entomology, 6, 285-294. https://doi.org/10.4236/ae.2018.64021

Received: September 16, 2018

Accepted: October 28, 2018

Published: October 31, 2018

Copyright (ㅇ 2018 by authors and Scientific Research Publishing Inc. This work is licensed under the Creative Commons Attribution International License (CC BY 4.0).

http://creativecommons.org/licenses/by/4.0/

\begin{abstract}
Hyalomma dromedarii ticks are important disease vectors to camels in the UAE and worldwide. Ticks can be identified using DNA-based techniques. In addition, such techniques could be utilized to study the intraspecific genetic diversity in tick populations. In this study, the genetic diversity of four $H$. dromedarii populations was investigated using mitochondrial cytochrome $\mathrm{c}$ oxidase subunit I (COI) gene and randomly amplified polymorphic DNA polymerase chain reaction (RAPD-PCR). The results showed that both of the aforementioned techniques produced similar grouping patterns. Moreover, they revealed that the four tick populations had high levels of genetic similarity. However, one population was slightly different from the three other populations. The current study demonstrated that $H$. dromedarii ticks in the UAE are very similar at the genetic level and that investigating more locations and screening larger numbers of ticks could reveal larger genetic differences.
\end{abstract}

\section{Keywords}

Camel Tick, Genetic Diversity, COI Gene, RAPD-PCR, Hyalomma dromedarii

\section{Introduction}

In order to successfully control tick-borne diseases, it is very important to develop good identification techniques, which enable successful separation of the 
different species of ticks. The RAPD-PCR is a molecular technique, which was used before the development of DNA sequencing. It is a good molecular technique, which is utilized for many years by taxonomists successfully and reliably [1] [2] [3]. However, with the advent of DNA sequencing new molecular identification techniques were developed by using segments of certain genes to provide good species identification. In a study [4], researchers developed a DNA barcoding system for the identification of Ixodid ticks using segment of the $16 \mathrm{~S}$ and COI genes. The identifications were successful, however it was mentioned that there were serious deficiencies in the information of $16 \mathrm{~S}$ and COI of some species of ticks, and thus additional research was needed to resolve this problem. In another study, medically important ticks were identified using a 658 base pair DNA barcode sequence from the COI gene [5]. Additionally, a genetic cluster analysis was performed on the barcode sequences and 26 Ixodid species were included. It was reported that DNA barcoding was a very good tool that clinicians could utilize to get the correct identification of tick specimens. To date, the use of a COI gene fragment is the standard marker for DNA barcoding. However, for COI, there are some limitations in its use with some species, and for some taxa [6]. In order to study their relative effectiveness, the COI, 16S ribosomal DNA (rDNA), nuclear ribosomal internal transcribed spacer 2 (ITS2) and $12 \mathrm{~S}$ rDNA were compared for tick species identification. The results showed that the intra-specific divergence of each marker was much lower than the inter-specific divergence. In addition, the aforementioned study indicated that COI was not significantly better than the other markers in terms of its rate of correct sequence identification. Nonetheless, COI should remain as the first choice for tick species identification. Moreover, a study reported that the DNA barcoding was successfully used in tick identification and that the K2P distances in most interspecific divergences exceeded $8 \%$, while intraspecific distances were usually lower than 2\% [7]. However, the study indicated that more research was required to reveal ambiguous species delimitation in some problematic genera. But is should be mentioned that there is a chance for some misidentification when using DNA-based techniques. A study was conducted as a comparative test of identification of ticks occurring in Western Europe and Northern Africa. There was a sizable misidentification rate and thus the recommendation was that a combination of certain genes may adequately identify the target species of ticks [8].

Ticks are vectors to many pathogens and parasites, which can cause serious diseases to human, domestic animal and wildlife [9] [10]. In different parts of the world such as North Africa, Southern Europe, Middle East, Central Asia and China Hyalomma (Acari: Ixodidae) ticks are common and may transmit Crimean-Congo hemorrhagic fever (CCHF), rickettsiae and tropical theileriosis [11] [12]. One of the potentially important parasites to domestic animal health in the Middle East is Theileria annulata because it infects cattle and is transmitted by Hyalomma spp. [12] [13] [14]. Ornithodoros savignyi collected from camels in Egypt was reported to harbor Rickettsia-like microorganisms [15]. 
Anaplasma marginale, Coxiella burnetii, Rickettsia aeschlim were reported from Egypt, Somalia and Nigeria from several Hyalomma spp. including $H$. dromedarii Koch, 1844 [12] [16]. Seventeen species of the genus Rickettsia are categorized within the Spotted Fever Group (SFG) rickettsiae [17] and several have been documented in the Middle East, although some reports are considered questionable [12]. In the UAE, a spotted fever group Rickettsia sp. and Theileria annulata were recorded for the first time in $H$. dromedarii ticks in the country [18]. Also, a Coxiella-like endosymbiont was reported in argasid ticks (Ornithodoros muesebecki) from a Socotra Cormorant colony in Umm Al Quwain, United Arab Emirates [19].

The aim of this study was to investigate the intraspecific genetic diversity of four $H$. dromedarii tick populations from the UAE.

\section{Materials and Methods}

\subsection{Tick Collection}

Camel ticks, $H$. dromedarii, Figure 1 were collected manually from the infested animals. Collected ticks were kept in $50-\mathrm{mL}$ plastic tubes inside a $-80^{\circ} \mathrm{C}$ freezer. Ticks were collected from four locations: Falaj Al-Muaalla, Bayata, Al-Wagan, and Samha. Falaj Al-Mualla and Bayata are located in the Emirate of Umm Al-Qaiwain while Al-Wagan and Al-Samha are in the Emirate of Abu Dhabi. Twelve ticks were collected per location.

\subsection{DNA Extraction and PCR}

Genomic DNA of camel ticks was extracted from tick legs using a Maxwell 16 Tissue DNA purification kit (Promega, USA) following the manufacturer's protocol. For the mitochondrial cytochrome $\mathrm{c}$ oxidase subunit I gene (COI) the following primer pair was used FishF1:

5'-TCAACCAACCACAAAGACATTGGCAC-3' and

FishR1: 5'-TAGACTTCTGGGTGGCCAAAGAATCA-3'. The template DNA was amplified in a $25 \mu \mathrm{l}$ reaction mixture containing $50 \mathrm{ng}$ of mite DNA, 10 pmol of each primer pair, and $12.5 \mu \mathrm{l} 2 \mathrm{x}$ PCR Master Mix (Promega, USA). Reaction mixtures were preheated at $95^{\circ} \mathrm{C}$ for $5 \mathrm{~min}$. Amplifications were carried out for 35 cycles $\left(95^{\circ} \mathrm{C}\right.$ for $30 \mathrm{~s}, 58^{\circ} \mathrm{C}$ for $30 \mathrm{~s}$, and $72^{\circ} \mathrm{C}$ for $1 \mathrm{~min}$ (final extension cycle at $72^{\circ} \mathrm{C}$ for $5 \mathrm{~min}$ ) in a Swift MaxPro thermocycler (ESCO, Singapore). For the RAPD-PCR assay, a set of 13 decamer primers (Operon Technologies, USA) were used. The sequences of these primers are presented in Table 1. These primers were selected as they generated consistent and reproducible RAPD banding patterns on the agarose gel. HotStarTaq ${ }^{\text {mi }}$ Master Mix kit (Qiagen, USA) was used for PCR reactions. The reaction mixture included $20 \mathrm{ng}$ template DNA, 12.5 $\mu \mathrm{L}$ HotStarTaq ${ }^{\text {tix }}$ Master Mix, and $50 \mathrm{pM}$ primer in a total volume of $25 \mu \mathrm{L}$. The program of thermal cycling was as follows: initial activation step at $95^{\circ} \mathrm{C}$ for $5 \mathrm{~min}$ followed by 40 cycles of $1 \mathrm{~min}$ at $94^{\circ} \mathrm{C}, 1 \mathrm{~min}$ at $36^{\circ} \mathrm{C}$ and $2 \mathrm{~min}$ at $72^{\circ} \mathrm{C}$, with a final extension step at $72^{\circ} \mathrm{C}$ for $5 \mathrm{~min}$. To detect contamination, a negative 


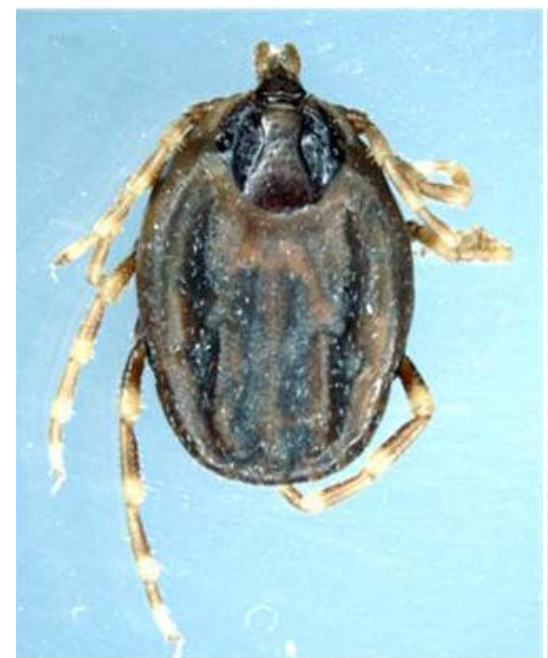

(a)

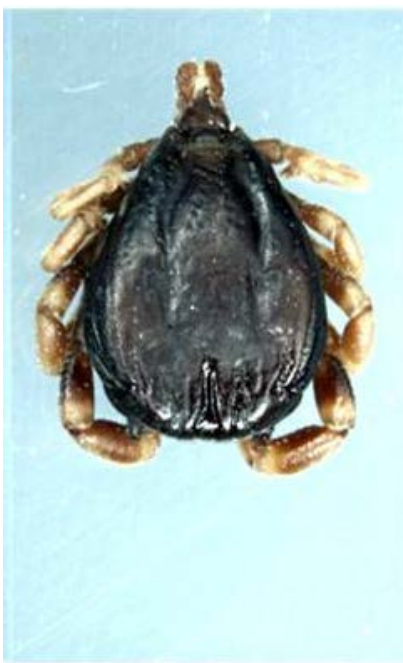

(b)

Figure 1. H. dromedarii adult female (a) and male (b). Ticks shriveled slightly after being stored in $70 \%$ ethanol.

Table 1. Random primers and number of generated bands of randomly amplified polymorphic DNA polymerase chain reaction (RAPD-PCR) in camel ticks.

\begin{tabular}{cccc}
\hline Primer name & Primer sequence 5'-3' & GC\% & No. of amplified bands \\
\hline OP-19A & CAAACGTCGG & 60 & 4 \\
OP-11A & CAATCGCCGT & 60 & 6 \\
OP-08A & GTGACGTAGG & 60 & 3 \\
OP-08B & GTCCACACGG & 70 & 10 \\
OP-02C & GTGAGGCGTC & 70 & 7 \\
OP-05C & GATGACCGCC & 70 & 3 \\
OP-10C & TGTCTGGGTG & 60 & 7 \\
OP-02D & GGACCCAACC & 70 & 3 \\
OP-08D & GTGTGCCCCA & 70 & 4 \\
OP-02E & GGTGCGGGAA & 70 & 9 \\
OP-10E & CACCAGGTGA & 60 & 6 \\
OP-09E & CTTCACCCGA & 60 & 6 \\
OP-17E & CTACTGCCGT & 60 & 3 \\
\hline
\end{tabular}

control, in which all PCR components except DNA template were included, was run in every experiment. In order to insure reproducibility of the individual bands, PCR reactions on the extracted DNA were conducted at least three times. Amplification products were resolved by electrophoresis in a 1.5\% agarose gel, which was stained with ethidium bromide and photographed by UVDI gel documentation system (Major Science, Taiwan). 


\subsection{PCR Amplicon Sequencing and Analysis}

In the RAPD-PCR, the banding pattern produced by each primer on the agarose gel was scored as one or zero (one for the presence of a band and zero for the absence of the band). Band scores were analyzed using cluster analysis in SPSS statistical package (IBM SPSS Statistics for Windows). The PCR amplicons were purified by a PCR purification kit (Qiagen, Germany) following the manufacturer's protocol. The purified PCR amplicons were sequenced (Sanger sequencing) by Source Bioscience (Cambridge, UK). Bases with too low confidence calling levels were trimmed from the beginning and end of sequence. Sequences were submitted in the GenBank and received accession numbers. They were analyzed by NCBI BLAST (http://blast.ncbi.nlm.nih.gov/Blast.cgi) analysis tool. In addition, sequence alignments and tree generation were conducted in MEGA6 [20]. The percentage of replicate trees in which the associated taxa clustered together in the bootstrap test (1000 replicates) was shown next to the branches. Genetic distances were computed using the Kimura 2-parameter method and were in the units of the number of base substitutions per site.

\section{Results}

\subsection{RAPD-PCR}

The analysis of the banding patterns produced by the random primers revealed that the four $H$. dromedarii populations were grouped into four clusters Figure 2. In this grouping of cluster analysis, the Bayata, Al-Wagan, and Falaj Al-Mualla were closer to each other compared to Al-Samha.

\subsection{COI Gene}

The sequences of the PCR amplicons ( $\approx 600 \mathrm{bp}$ ) of the mitochondrial COI gene were submitted in the GenBanK and received accession numbers: Falaj Al-Mualla: MG188798, Bayata: MG188799, and Al-Samha: MG188800. The sequence of Al-Wagan KJ022631 was submitted already in the GenBank in a previous study [18].

The sequences of Falaj Al-Mualla, Al-Wagan, and Bayata were $100 \%$ similar to each other, while the one of Al-Samha was different from them in two nucleotides. The intra-specific divergence value was 0.002 . In the maximum-likelihood Figure 3 and neighbor-joining Figure 4 trees the four sequences appeared in cluster of $H$. dromedarii sequences in which the sequence of Al-Samha showed up with some distance from the other three sequences. There was $99 \%-100 \%$ similarity and $99 \%-100 \%$ sequence coverage between the four sequences of the UAE and other H. dromedarii sequences in the GenBank such as KT920181.1, AJ437061.1, and KY548842.1. The inter-specific divergence value was 0.091 .

\section{Discussion}

The RAPD-PCR is a little bit an older molecular technique compared to Sanger sequencing, yet it is a good tool in studying genetic diversity. In a study [21] scientists reviewed more than 9000 papers that used RAPD and reported that 


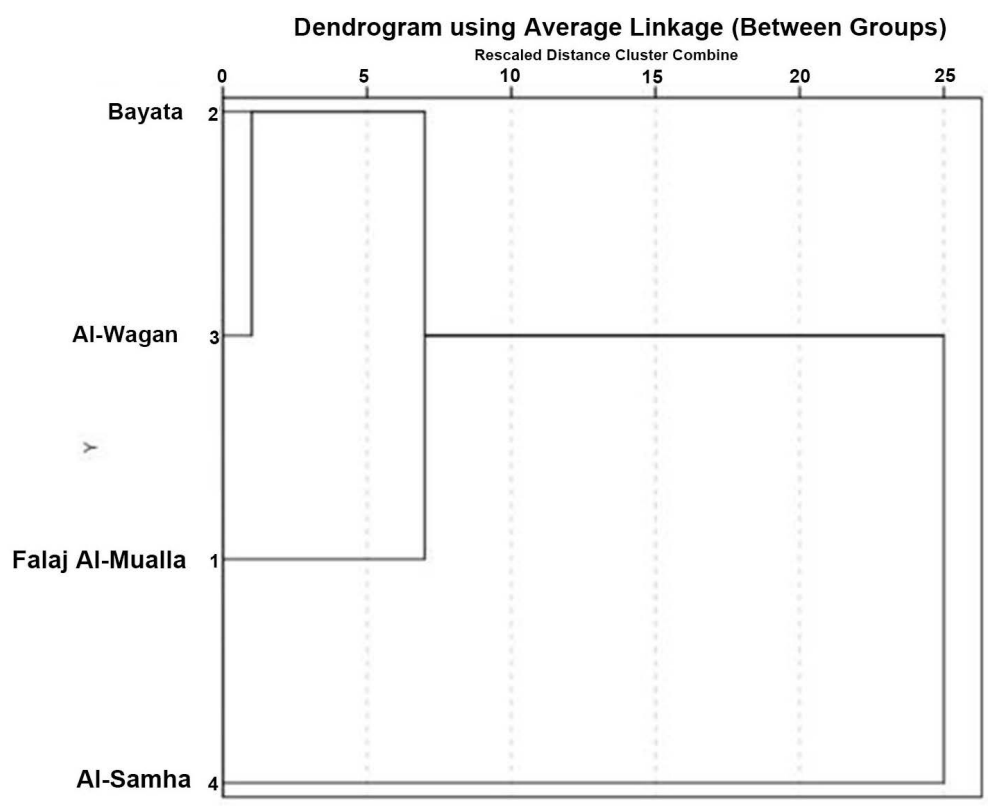

Figure 2. Dendogram of a cluster analysis showing three tick populations with higher DNA similarity versus a fourth population with less similarity (SPSS statistical package, IBM SPSS Statistics for Windows).

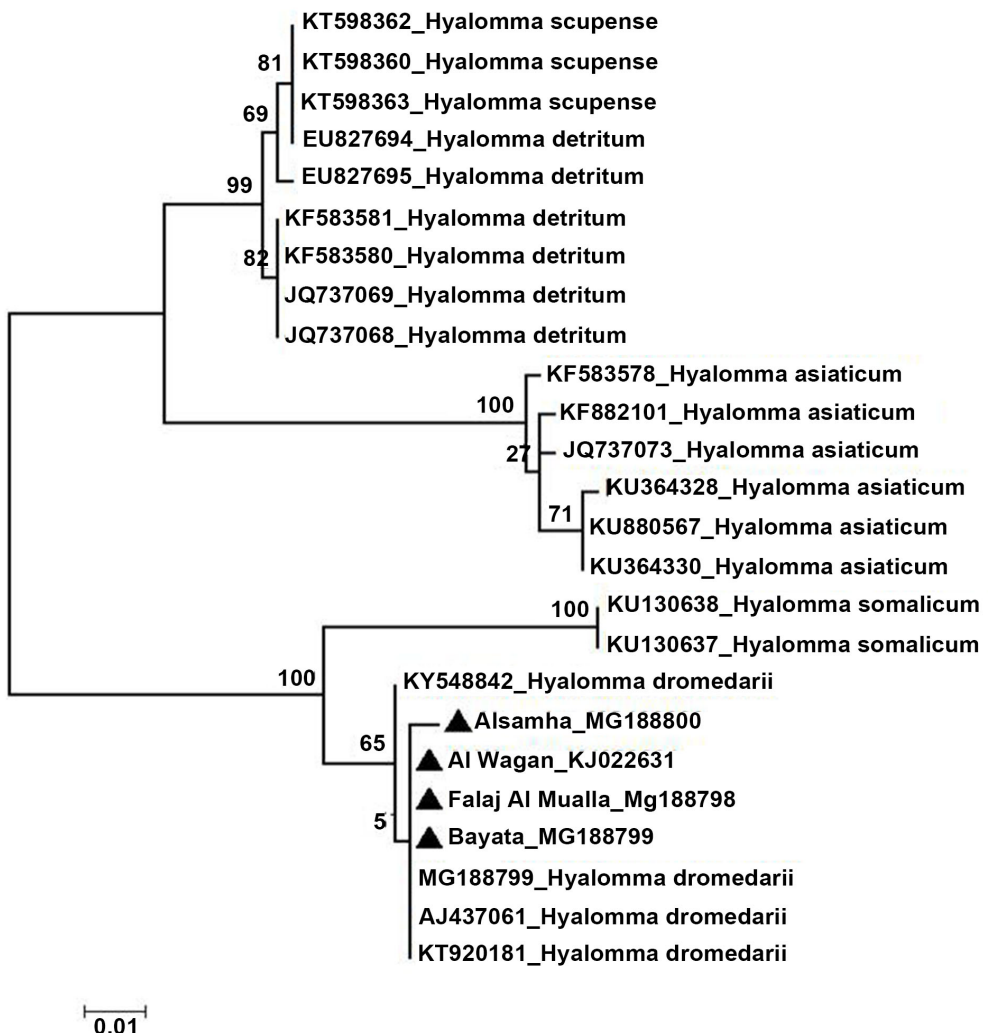

Figure 3. Maximum-likelihood tree constructed from nucleotide sequences (of the COI) of Hyalomma sp. based on Kimura 2-parameter. The Bootstrap branch support values were assessed with 1000 replicates. Falaj Al-Mualla (MG188798), Bayata (MG188799), Al-Samha (MG188800), and Al-Wagan (KJ022631) are four $H$. dromedarii sequences from the UAE. 


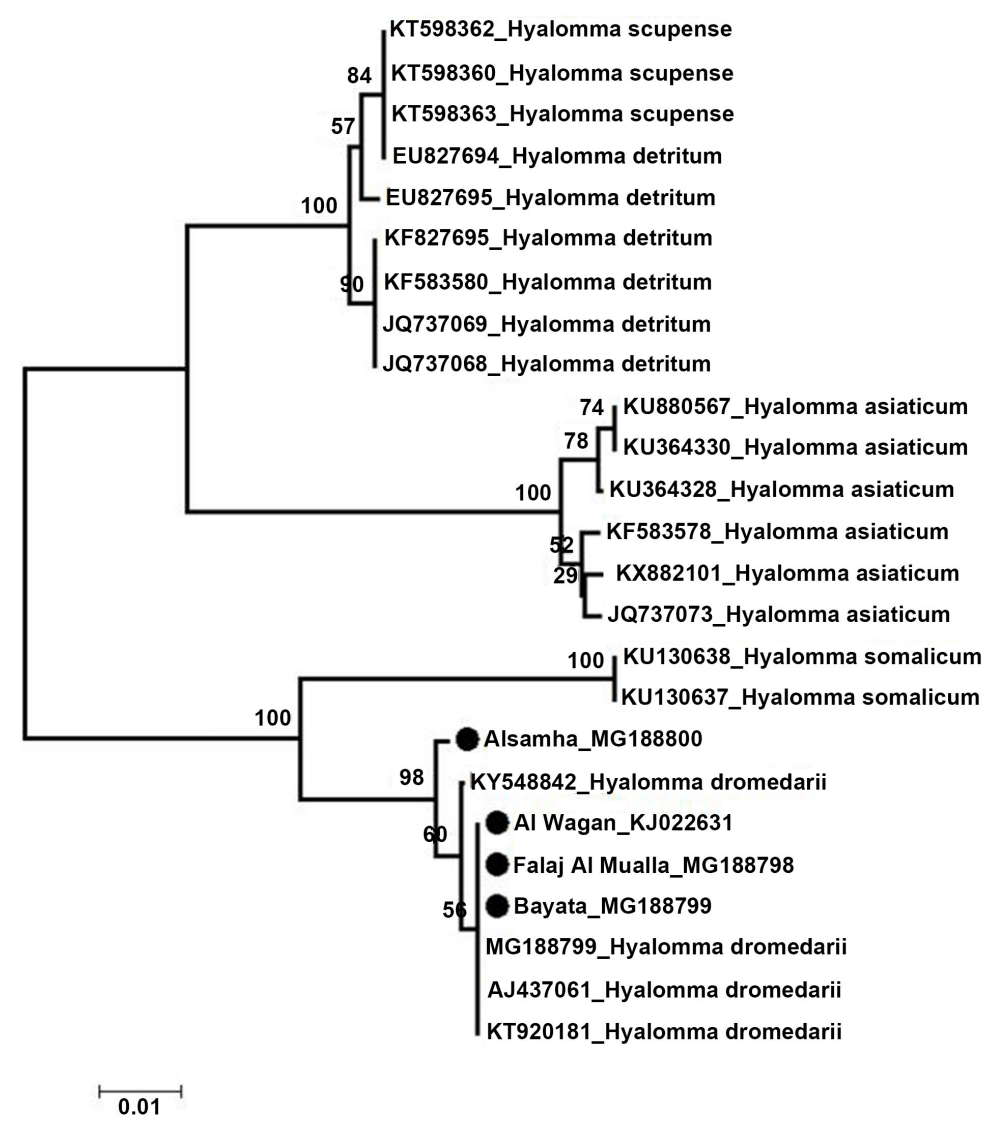

Figure 4. Neighbor-Joinig tree constructed from nucleotide sequences (of the COI) of Hyalomma sp. based on Kimura 2-parameter. The Bootstrap branch support values were assessed with 1000 replicates. Falaj Al-Mualla (MG188798), Bayata (MG188799), Al-Samha (MG188800), and Al-Wagan (KJ022631) are four $H$. dromedarii sequences from the UAE.

with proper optimization, RAPD is a reliable, sensitive and reproducible assay and has the potential to detect a wide range of DNA damage as well as mutations. The current study supported this finding and showed that the results of RAPD-PCR were in agreement with the results of the mitochondrial cytochrome c oxidase subunit I (COI) gene. Accordingly, three tick populations (Falaj Al-Mualla, Bayata, and Al-Wagan) were very similar, while Al-Samha had a small genetic difference as represented by the dendogram.

The use of DNA sequencing of the COI gene is a very reliable identification tool in animal species. The current study did not show high levels of genetic diversity among the $H$. dromedarii ticks collected from the four locations in the UAE. In part, this could be explained by the fact that the sampling was limited to a few areas of UAE in a relatively short period of time. Therefore, future studies should investigate more locations and screen larger numbers of ticks in order to possibly find bigger genetic differences. In this study, the four tick populations showed no intraspecific sequence difference except in one population (Al-Samha) there were two different nucleotides. Moreover, there was 99\% - 100\% similarity between the four sequences of and other $H$. dromedarii sequences in the Gen- 
Bank. The maximum-likelihood and neighbor-joining trees produced almost similar tree topologies and the branches were supported by high bootstrp values. The UAE tick sequences appeared in clusters of $H$. dromedarii on the trees. Other studies reported low genetic diversity when using COI gene. In a study on molecular identification of necrophagous Muscidae and Sarcophagidae fly species by COI, the data showed very low intraspecific sequence distances and species-level monophylies [22]. The COI gene was used successfully in conducting an assessment of genetic variability in dog ticks [23]. Studying genetic diversity in $H$. dromedarii tick populations is very important if some populations were different by harboring certain disease causing agents or by having acaricide resistance genes. Therefore, future studies should include as many geographical areas as possible in the UAE. This is very important in having successful disease and acaricide resistance management programs.

\section{Conclusion}

In conclusion, the current study demonstrated that $H$. dromedarii ticks in the UAE are very similar at the genetic level based on the four sampled locations. Also, the RAPD-PCR and the sequencing of the COI gene produced similar results. To our knowledge, the present study provides the first report of genetic diversity in $H$. dromedarii in the UAE.

\section{Acknowledgements}

We thank the Emirates Foundation in the UAE for providing the research grant from which this work was done.

\section{Conflicts of Interest}

The authors declare no conflicts of interest regarding the publication of this paper.

\section{References}

[1] Brown, R.J., Malcolm, C.A., Mason, P.L. and Nichols, R.A. (1997) Genetic Differentiation between and within Strains of the Saw-Toothed Beetle, Oryzaephilus surinamensis (Coleoptera: Silvanidae) at RAPD Loci. Insect Molecular Biology, 6, 285-289. https://doi.org/10.1046/j.1365-2583.1997.00184.x

[2] Black, W.C.I.V., Baer, C.F., Antolin, M.F. and DuTeau, N.M. (2001) Population Genomics: Genome-Wide Sampling of Insect Populations. Annual Review of Entomology, 46, 441-469. https://doi.org/10.1146/annurev.ento.46.1.441

[3] Barman, H.K., Barat, A., Yadav, B.M., Banerjee, S., Meher, P.K., Reddy, P.V.G.K. and Jana, R.K. (2003) Genetic Variation between Four Species of Indian Major Carps as Revealed by Random Amplified Polymorphic DNA Assay. Aquaculture, 217, 115-123. https://doi.org/10.1016/S0044-8486(02)00357-5

[4] Lv, J., Wu, S., Zhang, Y., Zhang, T., Feng, C., Jia, G. and Lin, X. (2014) Development of a DNA Barcoding System for the Ixodida (Acari: Ixodida). Mitochondrial $D N A, 25,142-149$. https://doi.org/10.3109/19401736.2013.792052

[5] Ondrejicka, D.A., Morey, K.C. and Hanner, R.H. (2017) DNA Barcodes Identify 
Medically Important Tick Species in Canada. Genome, 60, 74-84. https://doi.org/10.1139/gen-2015-0179

[6] Lv, J., Wu, S., Zhang, Y., Chen, Y., Feng, C., Yuan, X., Jia, G., Deng, J., Wang, C., Wang, Q., Mei, L. and Lin, X. (2014) Assessment of Four DNA Fragments (COI, 16S rDNA, ITS2, 12S rDNA) for Species Identification of the Ixodida (Acari: Ixodida). Parasites \& Vectors, 7, 1-11. https://doi.org/10.1186/1756-3305-7-93

[7] Zhang, L.R. and Zhang, B. (2014) Prospects of Using DNA Barcoding for species Identification and Evaluation of the Accuracy of Sequence Databases for Ticks (Acari: Ixodida). Ticks and Tick-Borne Diseases, 5, 352-358. https://doi.org/10.1016/j.ttbdis.2014.01.001

[8] Estrada-Peña, A., D’Amico, G., Palomar, A.M., Dupraz, M., Fonville, M., Heylen, D., Habela, M.A., Hornok, S., Lempereur, L., Madder, M., Núncio, M.S., Otranto, D., Pfaffle, M., Plantard, O., Santos-Silva, M.M., Sprong, H., Vatansever, Z., Vial, L. and Mihalca, A.D. (2017) A Comparative Test of Ixodid Tick Identification by a Network of European Researchers. Ticks and Tick-Borne Diseases, 8, 540-546. https://doi.org/10.1016/j.ttbdis.2017.03.001

[9] Al Khalifa, M.S., Diab, F.M., Al-Asgah, N.A., Hussein, H.S. and Khalil, G.A. (2006) Ticks (Acari: Argasidae, Ixodidae) Recorded in Wild Animals in Saudi Arabia. Fauna of Arabia, 2, 225-232.

[10] Diab, F.M., Al-Khalifa, M.S., Al-Asgah, N.A., Hussein, H.S. and Khalil, G.A. (2006) Ticks (Acari: Argasidae, Ixodidae) Infesting Livestock in Saudi Arabia. Fauna of Arabia, 2, 233-243.

[11] Wernery, U. and Kaaden, O.R. (2002) Infectious Diseases of Camelids. Blackwell Science, Vienna. https://doi.org/10.1002/9780470699058

[12] Aktas, M., Dumanli, N. and Angin, M. (2004) Cattle Infestation by Hyalomma Ticks and Prevalence of Theileria in Hyalomma Species in the East of Turkey. Veterinary Parasitology, 119, 1-8. https://doi.org/10.1016/j.vetpar.2003.10.013

[13] Hussein, H.S., Al-Asgah, N.A., Al-Khalifa, M.S. and Diab, F.M. (1991) The Blood Parasites of Indigenous Livestock in Saudi Arabia. Arab Gulf Journal of Scientific Research, 9, 143-160.

[14] De Kok, J.B., d'Oliveira, C. and Jongejan, F. (1993) Detection of the Protozoan Parasite Theileria annulata in Hyalomma Ticks by the Polymerase Chain Reaction. Experimental \& Applied Acarology, 17, 839-846. https://doi.org/10.1007/BF00225857

[15] Roshdy, M.A. (1968) A Rickettsia-Like Microorganism in the Tick Ornithodoros savignyi: Observations on Its Structure and Distribution in the Tissues of the Tick. Journal of Invertebrate Pathology, 11, 155-169. https://doi.org/10.1016/0022-2011(68)90146-8

[16] Loftis, A.D., Reeves, W.K., Szumlas, D.E., Abbassy, M.M., Helmy, I.M., Moriarity, J.R. and Dasch, G.A. (2006) Surveillance of Egyptian Fleas for Agents of Public Health Significance: Anaplasma, Bartonella, Coxiella, Ehrlichia, Rickettsia, and Yersinia pestis. The American Journal of Tropical Medicine and Hygiene, 75, 41-48. https://doi.org/10.4269/ajtmh.2006.75.41

[17] Hackstadt, T. (1996) The Biology of Rickettsiae. Infectious Agents and Disease, 5, 127-143.

[18] Al-Deeb, M.A., Muzaffar, S.B., Abu Zeid, Y., Enan, M.R. and Karim, S. (2015) First Record and Prevalence of Rickettsia sp. and Theileria annulata in Hyalomma dromedarii (Acari: Ixodidae) Ticks in the UAE. Florida Entomologist, 98, 135-139. https://doi.org/10.1653/024.098.0123 
[19] Al-Deeb, M.A., Frangoulidis, D., Walter, M.C., Kömpf, D., Fischer, S.F., Petney, T. and Bin Muzaffar, S. (2016) Coxiella-Like Endosymbiont in Argasid Ticks (Ornithodoros muesebecki) from a Socotra Cormorant Colony in Umm Al Quwain, United Arab Emirates. Ticks and Tick-Borne Diseases, 7, 166-171.

https://doi.org/10.1016/j.ttbdis.2015.10.012

[20] Tamura, K., Stecher, G., Peterson, D., Filipski, A. and Kumar, S. (2013) MEGA6: Molecular Evolutionary Genetics Analysis Version 6.0. Mol. Evolutionary Biology, 30, 2725-2729. https://doi.org/10.1093/molbev/mst197

[21] Atienzar, F.A. and Awadhesh, J.N. (2006) The Random Amplified Polymorphic DNA (RAPD) Assay and Related Techniques Applied to Genotoxicity and Carcinogenesis Studies: A Critical Review. Mutation Research, 613, 76-102.

https://doi.org/10.1016/j.mrrev.2006.06.001

[22] Kim, Y.H., Shin, S.E., Ham, C.S., S.Y., Kim, Ko, K.S., Jo, T.H., Son, G.H., Park, S.H. and Hwang, J.J. (2014) Molecular Identification of Necrophagous Muscidae and Sarcophagidae Fly Species Collected in Korea by Mitochondrial Cytochrome c Oxidase Subunit I Nucleotide Sequences. The Scientific World Journal, 2014, 1-9.

[23] Otranto, D., Latrofa, M.S., Brianti, E., Annoscia, G., Parisi, A., Dantas-Torres, F., Bain, O. and Gasser, R.B. (2012) An Assessment of Genetic Variability in the Mitochondrial Cytochrome c Oxidase Subunit 1 Gene of Cercopithifilaria sp. (Spirurida, Onchocercidae) from Dog and Rhipicephalus sanguineus Populations. Molecular and Cellular Probes, 26, 81-89. https://doi.org/10.1016/j.mcp.2011.12.002 